A. Bahri

\title{
Linking numbers in contact form geometry, with an application to the computation of the intersection operator for the first contact form of J. Gonzalo and F. Varela
}

\author{
To the memory of Leon Ehrenpreis
}

Received: 23 July 2013 / Accepted: 5 November 2013 / Published online: 19 February 2014

(c) The Author(s) 2014. This article is published with open access at Springerlink.com

\begin{abstract}
We establish in what follows the fact that the linking number between two periodic orbits does not decrease along the decreasing flow-lines of a suitable pseudo-gradient for the action functional of the variational problem $\left(J, C_{\beta}\right)$ and its extension $\left(J_{\infty}, \cup \Gamma_{2 k}\right),[2-4]$. This is used to compute almost explicitly the value of the intersection operator for periodic orbits for the case of the first exotic contact form of J. Gonzalo and F. Varela [8] on $S^{3}$.
\end{abstract}

Mathematics Subject Classification $37 \mathrm{~J} 45 \cdot 37 \mathrm{~J} 55 \cdot 53 \mathrm{D} 10 \cdot 55 \mathrm{~N} 99 \cdot 58 \mathrm{E} 10$

الملخص

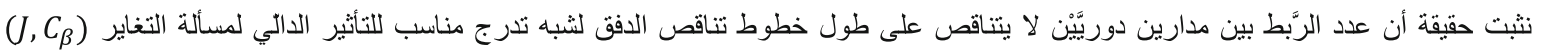

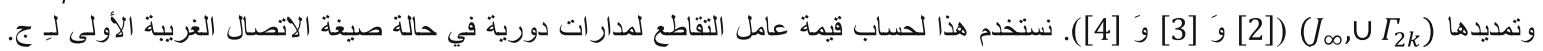

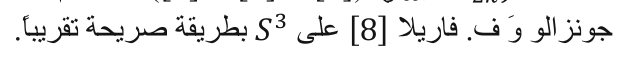

\section{Introduction}

Let $M^{3}$ be a three dimensional closed and orientable manifold with vanishing $\pi_{2}(M)$, e.g., $M=S^{3}$. Let $\alpha$ be a given contact form on $M^{3}$.

We assume for simplicity that ker $\alpha \longrightarrow M^{3}$ is trivial and we find therefore two independent non-zero vector-fields $v$ and $\bar{w}$ in $\operatorname{ker} \alpha$ such that $d \alpha(v, \bar{w})=1 . \xi$ is the Reeb vector-field of $\alpha$.

We consider the loop space $H^{1}\left(S^{1}, M\right)$ and the functional $J(x)=\int_{0}^{1} \alpha_{x}(\dot{x}) d t$ for $x \in H^{1}\left(S^{1}, M\right)$.

$\dot{x}$ reads as $a \xi+b v+c \bar{w}$. If $z \in T_{x} H^{1}\left(S^{1}, M\right)$ reads:

$$
z=\lambda \xi+\mu v+\eta w, \lambda, \mu, \eta \in H^{1}\left(S^{1}, \mathbb{R}\right)
$$

then,

$$
J^{\prime}(x) \cdot z=\int_{0}^{1}(c \mu-b \eta) d t
$$

A. Bahri ( $\varangle)$

Department of Mathematics, Rutgers, The State University of New Jersey,

110 Frelinghuysen Road, Piscataway, NJ 08854-8019, USA

E-mail: abahri@math.rutgers.edu 
We therefore recognize the "decreasing pseudo-gradient" for $J$

$$
z=-c w+b v=-“ \mathbf{J} \dot{x} "
$$

$z=-c v+b w$ is quite convenient on $H^{1}\left(S^{1}, M\right)$, but we are going to make it more particular:

We introduce as in $[1,3,4]$ a generalization of the Legendre duality derived under the assumption:

$$
\text { (A) } \beta=d \alpha(v, .)
$$

is a contact form with the same orientation than $\alpha$.

We will say that a contact form $\alpha$ of a given contact structure is $v$-convex if it verifies $(A)$ for a suitable $v$ in $\operatorname{ker} \alpha$.

$(A)$ is verified by convex Hamiltonians of $\mathbb{R}^{2 n}$ (and their associated contact forms, with $v$ a vector-field in their kernel defining a Hopf fibration). It is also verified [6] for some contact forms of the first exotic (thereby) over-twisted contact structure of J. Gonzalo and F. Varela [8], with $v$ the vector-field of Vittorio Martino [6].

Under $(A)$, we can restrict the variations of $J$ so that they take place in

$$
C_{\beta}=\left\{x \in H^{1}\left(S^{1}, M\right) ; \beta_{x}(\dot{x})=0, \alpha_{x}(\dot{x})=C \gtrless 0\right\}
$$

$C$ is not prescribed.

$\dot{x}$ then reads

$$
\dot{x}=a \xi+b v
$$

If $z=\lambda \xi+\mu v+\eta \bar{w}$ belongs to $T_{x} C_{\beta}$, then

$$
J^{\prime}(x) \cdot z=-\int_{0}^{1} b \eta d t
$$

In view of this formula, there is a "natural pseudo-gradient" that can be derived by taking $\eta=b$ in the formula above. It is tempting to write then that $z=b \bar{w}$. However, $z \in T_{x} C_{\beta}$ and therefore $z$ has a more complicated form. If $w$ is the contact vector-field of $\beta$ and $\alpha(w)=\bar{\mu}$, then $[3,4]$

$$
z=\left(\int_{0}^{t} b^{2}-t \int_{0}^{1} b^{2}-\bar{\mu} b\right) \xi+\frac{\dot{b}+b\left(\int_{0}^{t} b^{2}-t \int_{0}^{1} b^{2}-\bar{\mu}\right)}{a} v+b w
$$

The evolution equation $\frac{\partial x}{\partial s}=z(x), x(0)=x_{0} \in C_{\beta}$ is very close to the mean or normal curvature equation on one dimensional curves.

This flow has several remarkable geometric properties $[3,4,6]$. One of them is that the linking of two curves [assume that they are both contractible or that $\pi_{1}(M)=0$ so that their linking is defined then without ambiguity since $\pi_{2}(M)=0$ ] under $J$-decreasing evolution through the flow of $z$ never decreases, see [6], Lemma 13 for an account of this property.

However, this flow does have several "undesired" blow-ups (in [3], a flow that "corrects" the defects of the flow described above is defined and studied) and it is therefore difficult to define a homology related to the periodic orbits of the Reeb vector-field $\xi$ with this "pseudo-gradient".

In a general framework, which includes cases of exotic contact forms and structures, there is no obvious need to limit ourselves to the natural $z$ that we found above. Freeing ourselves of any special form, we seek for a general decreasing "pseudo-gradient" for the functional $J$ on $C_{\beta}$.

We have throughout our work, see [3-5], in particular another "pseudo-gradient" $Z$ that blows up only along the stratified set $\cup \Gamma_{2 k}$, where $\Gamma_{2 k}=$ \{curves made of $k$-pieces of $\xi$-orbits, alternating with $k$-pieces of $\pm v$-orbits\}. 
In our constructions [3-5], $Z$ has important properties: along its (semi)-flow-lines, the number of zeros of $b$, the $v$-component of $\dot{x}$ that is the tangent vector to the curve $x$ under deformation, never increases; the $L^{1}$-norm of $b$ is bounded; all (semi)-flow-lines end either at critical points (periodic orbits) or critical points at infinity ([3-5]). In addition, see [5], Proposition 2.1, p 469 and the first section of [7], the behavior of $Z$ in the vicinity of periodic orbits can be prescribed.

$Z$ has one additional property, studied in [6], Sect. 7 and after, that is summarized in Lemma 13 of [6]. Namely, given a decreasing flow-line $C_{S}$ originating at a periodic orbit of the Reeb vector-field $\xi$ and another periodic orbit $O^{\prime}$, with $O^{\prime}$ contractible in $M$ so that the linking number with $O^{\prime}$ is well-defined, the linking number $l k\left(C_{s}, O^{\prime}\right)$ only increases with $s$. This is related to the property that, for our flow $Z, b \eta \geq 0$, where $\eta$ is the $w$-component of $Z$.

However, as soon as we extend $Z$ to the stratified set $\cup \Gamma_{2 k}$, this property no longer holds if $Z$ is just an arbitrary pseudo-gradient for the extended functional $J_{\infty}$, see [3] p 203, [4], p 6, on this set.

We prove in this paper two results in this regard. One is theoretical, the other one is an application of this theoretical result to the case of the first exotic contact form of J. Gonzalo and F. Varela [8]:

Theorem 1.1 Z can be extended into a "pseudo-gradient" of $J_{\infty}$ on $\cup \Gamma_{2 k}$ so that, for any decreasing flow-line $C_{s}$ of this extended $Z$ originating and ending at periodic orbits of $\xi$ with a difference of indexes equal to 1 , $l k\left(C_{s}, O^{\prime}\right)$ never decreases with $s$.

The proof of Theorem 1.1 is based on an extension of the results of [6], Sect. 3. Namely, we establish throughout Sect. 2 of the present paper that, if this linking is to decrease, then we can arrange so that the flow-line $C_{\mathrm{s}}$ does not reach another periodic orbit (under the assumption that the difference of Morse indexes is 1$)$.

We then move to study the intersection operator $\partial_{\text {per }}$, see [6] and [7]-it is the intersection operator for the extended $Z$ restricted to periodic orbits-, with the help of Theorem 1.1, for the first exotic contact form $\alpha_{1}$ of J. Gonzalo and F. Varela [8].

$$
\alpha_{1} \text { reads as: } \alpha_{1}=-\cos \left(\frac{\pi}{4}+\pi r_{2}\right)\left(x_{2} d x_{1}-x_{1} d x_{2}\right)-\sin \left(\frac{\pi}{4}+\pi r_{2}\right)\left(x_{4} d x_{3}-x_{3} d x_{4}\right)
$$

$S^{3}$ is the standard sphere $\left\{\left(x_{1}, x_{2}, x_{3}, x_{4}\right) \in R^{4}, x_{1}^{2}+x_{2}^{2}+x_{3}^{2}+x_{4}^{2}=1\right\}$.

We are able with the use of this linking property to give restrictions on a periodic orbit $O$, given $O^{\prime \prime}$, so that they are related by the intersection operator computed on periodic orbits for this flow: $O^{\prime \prime} \in \partial_{\text {per }}(O)$. In order to describe the precise result, we recall that every periodic orbit in this framework, see Sect. 7 of [6], has two associated integers $(p, q) . p$ and $q$ are the counter-clockwise rotations in the planes $\left(x_{3}, x_{4}\right)$ and $\left(x_{1}, x_{2}\right)$, respectively.

Let $A=\cos \left(\frac{\pi}{4}+\pi r_{2}\right), B=\sin \left(\frac{\pi}{4}+\pi r_{2}\right)$

$\tilde{A}=A+\pi r_{1} B, \tilde{B}=B+\pi r_{2} A$. $p$ has the sign of $\tilde{A}$ and $q$ has the sign of $\tilde{B}$, see [6], Sect. 7 .

Let $(p, q)$ be associated to $O$ and $\left(p^{\prime \prime}, q^{\prime \prime}\right)$ be associated to $O^{\prime \prime}$. Then,

Theorem 1.2 (i) If $O^{\prime \prime} \in \partial_{\text {per }}(O)$, then either $p$ and $p^{\prime}$ are negative (then $q$ and $q^{\prime}$ are also negative) or $q$ and $q^{\prime}$ are positive (then $p$ and $p^{\prime}$ are also positive). In the first case, $p=p^{\prime}, q^{\prime}=q+1$. In the second case, $q=q^{\prime}, p=p^{\prime}+1$. (ii) the homology related to $\partial_{p e r}$ for the first exotic contact form of $J$. Gonzalo and $F$. Varela [8] has at least two generators for a sequence of indexes tending to $\infty$.

Section 2 of this paper is devoted to the proof of Theorem 1.1, whereas Sect. 3 of this paper is related to the proof of Theorem 1.2.

\section{Linking numbers of periodic orbits for Reeb vector-fields and decreasing flow-lines for the action functional on $C_{\beta}$}

Along the flow-lines of our decreasing pseudo-gradient $Z$, at a curve $x$ of $C_{\beta}$, with $\dot{x}=a \xi+b v$, as long as the condition $b \eta \geq 0$ is satisfied- $\eta$ is the $w$-component of the pseudo-gradient $Z$ at $x$-, the linking number of a curve under decreasing deformation with a given periodic orbit cannot decrease. see [6], Lemma 13.

However, once our curves reach $\cup \Gamma_{2 s}$, the deformation does not verify the condition $b \eta \geq 0$ anymore, so that the linking number of a curve under decreasing deformation with a given periodic orbit might now decrease.

We claim that this might happen, but only with flow-lines that reach very precise curves that we describe in the sequel.

Let us consider a dominating periodic orbit $\mathrm{O}^{+}$and let us assume that a decreasing flow-line originating at this periodic orbit abuts at a curve $z$ of $\Gamma_{2 m}$ that "touches" another periodic orbit $O^{\prime}$. 
Now, the linking number can change, but we want to understand when it is that it has to change; that is when it is that we cannot define our flow so that this linking number does not change.

We claim that:

Proposition 2.1 If $z$ "touches" $O^{\prime}$ along one or more of its $\xi$-pieces and does not "touch" it elsewhere and if $O^{\prime}$ is hyperbolic, then the linking number of the curves under decreasing deformation with $O^{\prime}$ does not change through this "touching".

Furthermore, we claim that:

Proposition 2.2 Assume that the curve $z$ "touches" $O^{\prime}$ along at least one of its $\pm v$-pieces.

If the linking number of the curves under decreasing deformation with $O^{\prime}$ is forced to change at $z$, then $z$ $\left(z\right.$ is in $\left.\Gamma_{2 m}\right)$ is an isolated curve subject to $2 m$ independent conditions.

Proof of Proposition 2.1 The linking number of two curves in $S^{3}$ can be computed as the intersection number of a chain whose boundary is one of the curves with the other curve. Orientations are given according to domains.

Given a hyperbolic periodic orbit $O^{\prime}$, we may assume without loss of generality that it is of even index, see [7], Sect. 3; its stable and unstable manifolds from the point of view of dynamics, not from the variational point of view, are tangent to $\xi$. They have $O^{\prime}$ as a boundary (the index is even).

One of these two, e.g., $W_{u}^{d}\left(O^{\prime}\right)$-the upper index $d$ stands for dynamical, not variational- the dynamical unstable manifold of $O^{\prime}$ can be included as a part of a chain $c$ such that $\partial c=O^{\prime}$. It then follows that the intersection number cannot change through the $\xi$-pieces of $z$ : such a $\xi$-piece that touches $O^{\prime}$ will either, later, as the decreasing deformation proceeds, stay on "one side" of $c$; or it will cross at two points (for a given and "touching" $\xi$-piece), with opposite orientations $\left(W_{\mathrm{u}}^{\mathrm{d}}\left(O^{\prime}\right)\right.$ is embedded near $\left.O^{\prime}\right)$. Therefore, the linking number can change only if $z$ touches $O^{\prime}$ with one or more of its $\pm v$-jumps.

Proof of Proposition 2.2 Let us assume that $z$ touches $O^{\prime}$ with $s$ of its $\pm v$-jumps (counted with multiplicity; most of these "touchings" will involve at most one "touching" per $\pm v$-jump. Arguments adjust accordingly, with more precise studies, if there are $\pm v$-jumps of $z$ touching $O^{\prime}$ at multiple points).

The curve $z$ is then subject to $s$ conditions, namely that its $\pm v$-jumps "touch" $O^{\prime}$ at $s$ points to the least.

We would like to define a decreasing deformation, across $z$ that will not change the linking number. We consider the tangent space at $z$ to $\Gamma_{2 m}$ ( $z$ belongs to $\left.\Gamma_{2 m}\right) T_{z} \Gamma_{2 m}$ and, in this space, we look for all the tangent vectors that are along $\mathbb{R} \xi+\mathbb{R} v$ at these touching points.

This imposes $s$ conditions at these "touching" points.

If we assume that general position holds, we find then a sub-space $E$ of $T_{z} \Gamma_{2 m}$ of dimension $2 m-s$, along which these $s$-conditions are satisfied.

We then derive that if:

$$
\partial J(z) \cdot h \neq 0
$$

for some $h$ of $E$, then we can define a decreasing deformation that does not change the linking. We can then even perturb a bit $h$ into $h_{1}, h_{1}$ not in $E$ anymore, so that the curves under deformation do not touch $\bar{O}^{\prime}$ anymore, whereas the linking number does not decrease, in fact possibly increases. Our arguments can then proceed.

If not, $\partial J(z)_{\mid E}=0$. This yields $(2 m-s)$ conditions. These $(2 m-s)$ conditions, when combined with the $s$ conditions of "touching", yield $2 m$ conditions, which we claim, using Proposition 29, p 198 of [3] (observe that the perturbation of $v$, this can be seen in the proof of Proposition 29 of [3], can be completed on a piece of $v$-orbit that is "far" from the points of "touching") are independent.

We thus see that these $z \mathrm{~s}$ are isolated curves that can be thought of, subject to these $s$ "touching" conditions, to be critical points of $J_{\infty}$ (under these additional conditions).

Of course, these curves can be "bypassed" through a $J_{\infty}$-decreasing deformation; but the linking number with $O^{\prime}$ might decrease.

This will matter only if the flow-lines out of $z$ reach a periodic orbit of index equal to index $O^{+}-1$.

We invoke then the arguments of Sect. 3 of [7] and Sect. 11 of the same paper. These arguments imply, with very few modification involving the adjustment of the framework that the decreasing flow, out of $z$, can be defined so that all these flow-lines never abut at such a periodic orbit.

We then claim (Fig. 1): 
a

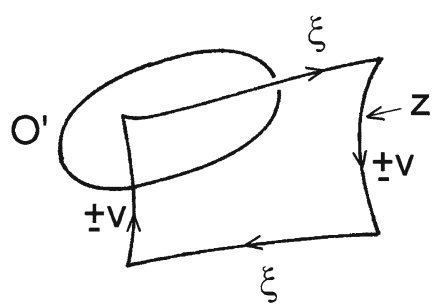

b

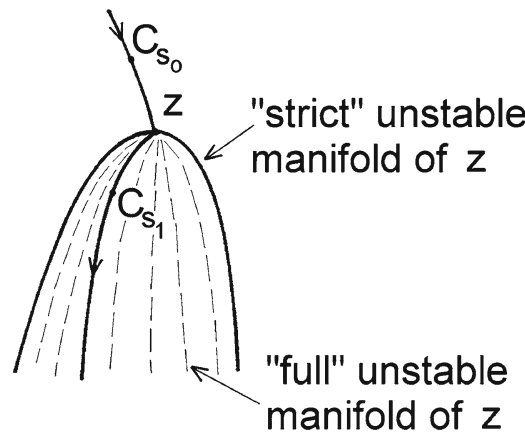

\section{The linking number $\mathrm{Ik}\left(\mathrm{C}_{\mathrm{s}}, \mathrm{O}^{\prime}\right)$ changes by \pm 1 at the crossing of $z$}

Fig. 1

Proposition 2.3 The decreasing flow can be defined so that, if $\mathrm{O}^{-}$is a periodic orbit dominated by $\mathrm{O}^{+}$, with a difference of Morse indexes equal to 1 between $O^{+}$and $O^{-}$, then the linking number of $O^{+}$with $O^{\prime}$ is less than or equal than the linking number of $O^{-}$with $O^{\prime}$.

Proof of Proposition 2.3 The decreasing flow is now defined along the flow-lines out of the special curves $z$. These flow-lines, by the arguments above, never reach a periodic orbit.

We can extend it so that, outside of a small neighborhood of these flow-lines, the flow does have the property that the linking number of the curves under deformation with $O^{\prime}$ never decreases. However, we also have other flow-lines that come from outside such a neighborhood, enter this neighborhood and then exit it. We thus may then find flow-lines that go to periodic orbits $O^{-}$, but along which the non-decreasing property of the linking number with $O^{\prime}$ or another periodic orbit fails.

Let us then observe, as pointed out above, that along the $W_{\mathrm{u}}(z) \mathrm{s}$, that is along the flow-lines out of the $z \mathrm{~s}$ encountered above, the non-decreasing property of this linking number will hold as long as these flow-lines do not reach some of these curves $z$ s.

Thus, if we remove small neighborhoods of the zs, all flow-lines do verify the non-decreasing property, even after completing the convex-combinations that are required in order to patch the flows over small chosen neighborhoods of the $W_{\mathrm{u}}(z) \mathrm{s}$ which we use to build our flow.

The flow-lines that are left must enter neighborhoods of the $z$ s, neighborhoods that are small as we wish. These flow-lines start at an ${O^{+}}^{\prime}$ and they have to go to one of these $z$ s.

Considering the flow-lines from this $O^{+^{\prime}}$ to such a $z$, let us take the first such $z$ encountered, these are not anymore in the neighborhoods of $W_{\mathrm{u}}(z)$ after we remove a neighborhood of $z$ itself that is as small as we wish. Indeed, these flow-lines are coming from "above", they are not originating at $z$.

It follows that they come to $W_{\mathrm{u}}(z)$ (all the unstable manifolds have to be understood as in [7]; transversality holds in the $\Gamma_{2 k} \mathrm{~s}$, but for the $H_{0}^{1}$-directions if we do not use "companions" as in [6]. However, even without the use of companions, Morse theory extends, see [7] for more details from above and enter into $W_{\mathrm{u}}(z)$ from above, just as flow-lines do as they come from above a critical point in the Morse Lemma: these flow-lines are part of $W_{\mathrm{s}}(z)$ (under the $s$ constraints of "touching" that are imposed on $z$ ). They enter therefore, as the flow in continued through $W_{\mathrm{u}}(z)\left(W_{\mathrm{u}}(z)\right.$ is not now the "strict" unstable manifold of $z$, that is its unstable manifold subject again to these $s$ constraints; it contains now the additional $J_{\infty}$-decreasing directions that might increase the linking), the core of the neighborhood of infinity used to patch the flows. This happens under the assumption that their index is equal to $\operatorname{dim} W_{\mathrm{u}}(z)$. Otherwise, their index is larger than the dimension 
a

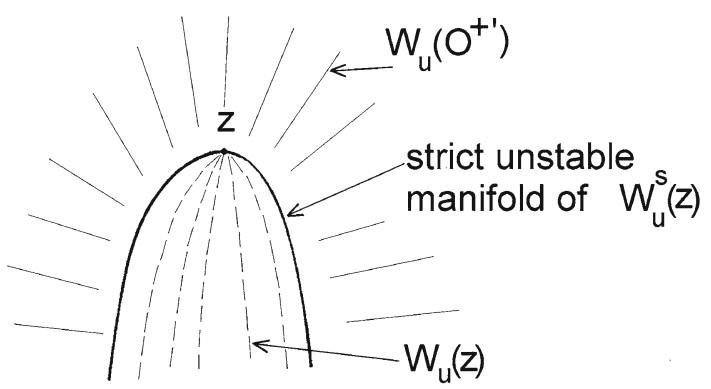

- If index $\mathrm{O}^{+{ }^{\prime}}=1+\operatorname{index} \mathrm{O}^{-}, \operatorname{dim} \mathrm{W}\left(\mathrm{O}^{+{ }^{\prime}}\right)=\operatorname{dim} \mathrm{W}_{\mathrm{u}}^{\mathrm{s}}(\mathrm{z})+1$, and all of $W_{u}\left(O^{+\prime}\right)$, near $z$, will continue with $W_{u}(z)$, therefore avoiding $\mathrm{O}^{-}$(see argument in text)

- If index $\mathrm{O}^{+1}>$ index $\mathrm{O}^{-}+1, \mathrm{O}^{-} \notin \partial_{\text {per }}\left(\mathrm{O}^{+\prime}\right)$

b

broken flow-line, due

z to patching (limit behavior

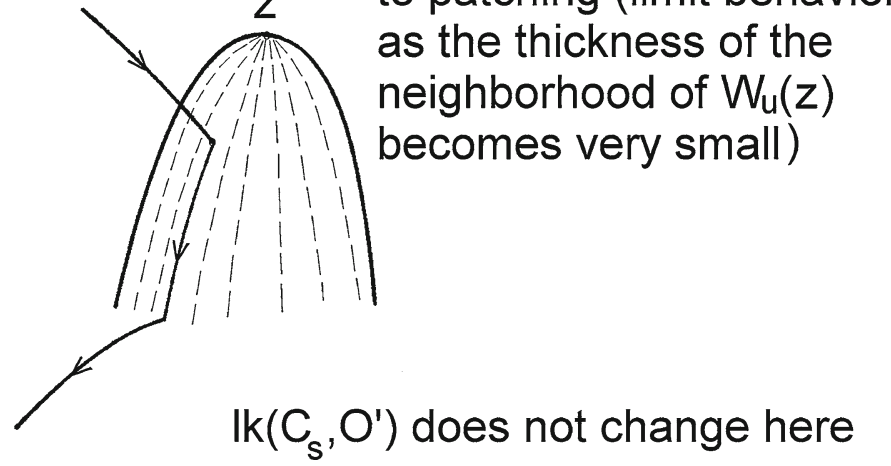

Fig. 2

of $W_{\mathrm{u}}(z)$ and, therefore, if a periodic orbit $O^{-}$is dominated by $W_{\mathrm{u}}(z)$ before perturbation, then this orbit is of index less than equal than $\operatorname{dim} W_{\mathrm{u}}(z)-1 \lesseqgtr$ index $O^{+\prime}-1$ and we are not concerned with $O^{-}$if the flow-lines originate at $O^{+\prime}$. The patching, over the perturbation that removes $O^{-}$from $\bar{W}_{\mathrm{u}}(z)$, can be completed without worrying about the flow-lines of this $W_{\mathrm{u}}\left(O^{+\prime}\right)$ near $W_{\mathrm{u}}(z)$. For the other $O^{+} \mathrm{s}$ that have an index equal to $\operatorname{dim} W_{\mathrm{u}}(z)$, their flow-lines, as explained above, enter the core of the neighborhood of infinity used to patch the flows. For this very reason, they are not involved in the patching, they are part of this extended $W_{\mathrm{u}}(z)$ and they never reach a periodic orbit $O^{-}$. The conclusion follows for this precise $O^{+}$and the flow-lines originating at $\mathrm{O}^{+}$. The argument can be extended to all the periodic orbits $\mathrm{O}^{+}$of this given index-they are in finite numberand, from there, by induction, extended to all the periodic orbits. The general conclusion follows.

\section{The operator $\partial_{\text {per }}$ for the first exotic contact form of J. Gonzalo and F. Varela [8]}

We study the operator $\partial_{\text {per }}$ which is the restriction of the intersection operator for our flow to the periodic orbits of $\xi$. We will focus here on the periodic orbits of $\xi_{0}$, the Reeb vector-field of the standard first exotic contact form of J. Gonzalo and F. Varela [8]. We will first study those involving the periodic orbits $O^{ \pm}$corresponding to $r_{1}=0\left(x_{1}=x_{2}=0\right)$ and to $r_{2}=0\left(x_{3}=x_{4}=0\right)$ in $S^{3}=\left\{\left(x_{1}, x_{2}, x_{3}, x_{4}\right) \in \mathbb{R}^{4} ; x_{1}^{2}+x_{2}^{2}+x_{3}^{2}+x_{4}^{2}=1\right\}$. Generalizations will follow easily.

We will use the conclusion of Proposition 2.1 stating that the linking number $l k\left(C_{s}, O^{\prime}\right)$ of a family of curves under decreasing deformation (deformation as we built it throughout [1-5], especially [3] and [4] and the present work) with a hyperbolic orbit $O^{\prime}$ never decreases.

Let us first assume that, with $O^{+}$being the iterate of order $\bar{p}_{1}$ of the periodic orbit of $\xi_{0}$ corresponding to $r_{1}=0\left(r_{2}=1\right)$, we have: 


$$
O_{3} \in \partial_{\mathrm{per}} O^{+}
$$

with $O_{3}$ a periodic orbit with $r_{1} \neq 0, r_{2} \neq 0$ and associated numbers, see [6], Sect. 7, $(p, q)=\left(\bar{p}_{3}, \bar{q}_{3}\right) ; p$ and $q$ are the counter-clockwise rotations in the planes $\left(x_{3}, x_{4}\right)$ and $\left(x_{1}, x_{2}\right)$, respectively.

Let $O_{2}$ be a periodic orbit with associated numbers $(p, q)=\left(\bar{p}_{2}, \bar{q}_{2}\right) . O_{2}$ is never taken to be neither $O^{+}$ nor $O^{-}$. It can always be taken to be a hyperbolic periodic orbit, of even index $2(p-q)$. It is then the "top index" periodic orbit, after perturbation of the circle of periodic orbits tangent to the $X_{0}$ vector-field, that has the associated rotations numbers $(p, q)$ in the planes $\left(x_{3}, x_{4}\right)$ and $\left(x_{1}, x_{2}\right)$ respectively, see [6], Sect. 2 for more details.

We then claim:

Proposition 3.1 $\bar{q}_{3}$ is a negative integer. This conclusion holds also if $\mathrm{O}_{3}=\mathrm{O}^{-}$.

Proof of Proposition 3.1 Assume that $\bar{q}_{3}$ is a positive integer. We choose $\mathrm{O}_{2}$ so that $r_{2}\left(\mathrm{O}_{2}\right) \lesseqgtr r_{2}\left(\mathrm{O}_{3}\right)$ and with $\bar{p}_{2}$ and $\bar{q}_{2}$ positive. We know, see [6], Sect. 7, after Corollary 1, that the number $p$ has the sign of $\tilde{A}=\cos \left(\frac{\pi}{4}+\pi r_{2}\right)+\pi r_{1} \sin \left(\frac{\pi}{4}+\pi r_{2}\right) ;$ and that $q$ has the sign of $\tilde{B}=\sin \left(\frac{\pi}{4}+\pi r_{2}\right)+\pi r_{2} \cos \left(\frac{\pi}{4}+\pi r_{2}\right)$. Therefore, using Fig. 3 below which gives the sign of various quantities:

this choice for $\mathrm{O}_{2}$ is possible if $\bar{q}_{3}$ is positive.

We then must have:

$$
l k\left(O^{+}, O_{2}\right) \leq l k\left(O_{3}, O_{2}\right)
$$

This yields, by Lemma 16 of [6]:

$$
-\bar{p}_{1} \bar{q}_{2} \leq-\bar{p}_{2} \bar{q}_{3}
$$

If $\bar{q}_{3}$ is positive, $-\bar{p}_{2} \bar{q}_{3}$ is negative, whereas $-\bar{p}_{1} \bar{q}_{2}$ is positive; a contradiction.

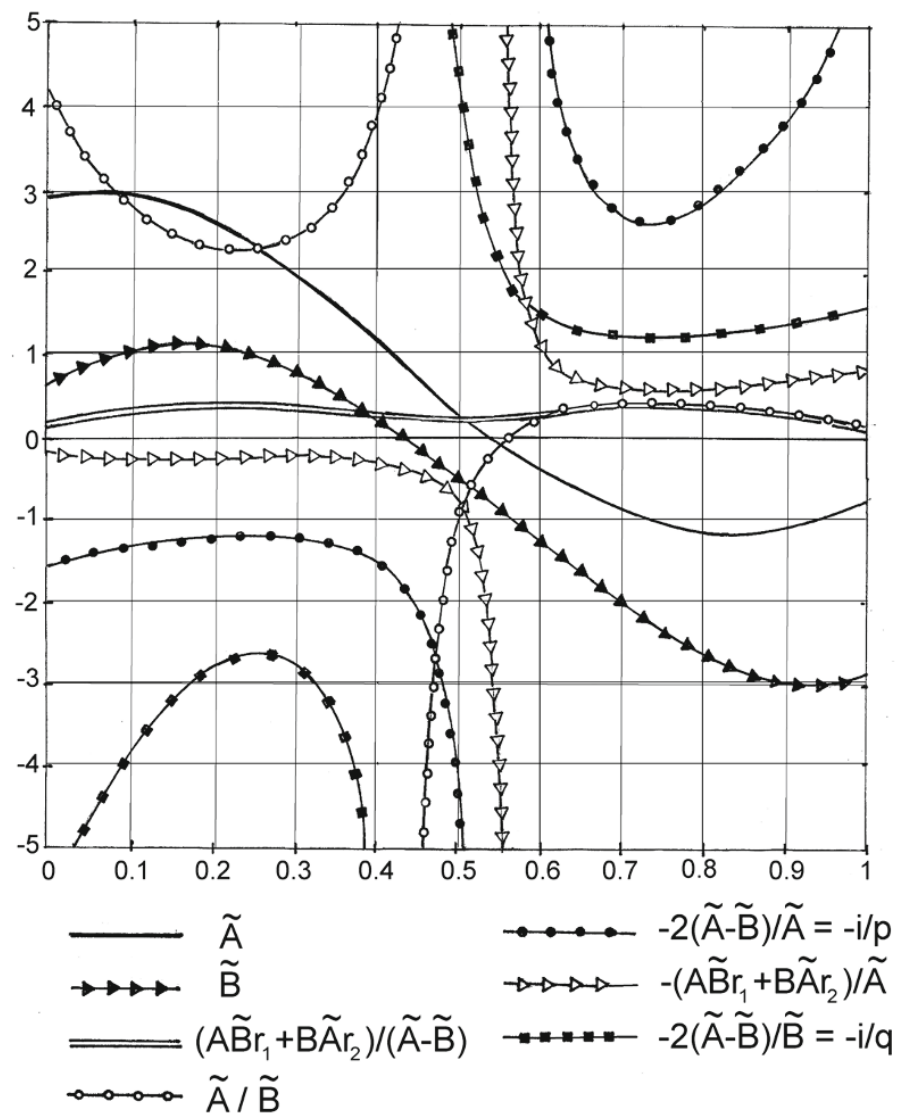

Fig. 3 
We need now to exclude that $\bar{q}_{3}$ is zero.

If $\bar{q}_{3}$ is zero, we choose $O_{2}$ such that

$$
0 \lesseqgtr r_{2}\left(O_{2}\right) \lesseqgtr r_{2}\left(O_{3}\right)
$$

We then have:

$$
-\bar{p}_{1} \bar{q}_{2} \leq-\bar{p}_{3} \bar{q}_{2}
$$

$\bar{q}_{2}$ is positive, see Fig. 3 above. $-\bar{p}_{1}$ is positive, whereas $-\bar{p}_{3}$ is negative, since $\tilde{A}$ is positive when $\tilde{B}$ is zero, see Fig. 3, above. The conclusion follows.

This Proposition extends into:

Proposition 3.2 Assume that $\mathrm{O}_{3}$ is as above and that $\mathrm{O}^{+} \in \partial_{\text {per }} \mathrm{O}_{3}$. Then $\bar{q}_{3}$ is a negative integer again. This conclusion holds also if $\mathrm{O}_{3}=\mathrm{O}^{-}$.

Proof of Proposition 3.2 Arguing as above, assuming that $\bar{q}_{3}$ is a non-negative integer, we now have:

$$
-\bar{p}_{1} \bar{q}_{2} \geq-\bar{p}_{2} \bar{q}_{3}
$$

We now take both $\bar{p}_{2}$ and $\bar{q}_{2}$ to be negative. This is possible and it is compatible with the requirement $r_{2}\left(\mathrm{O}_{2}\right) \geqslant r_{2}\left(\mathrm{O}_{3}\right)$, see Fig. 3 above.

$-\bar{p}_{1} \bar{q}_{2}$ is then negative, whereas $-\bar{p}_{2} \bar{q}_{3}$ is non-negative if $\bar{q}_{3}$ is negative; a contradiction again.

We thus conclude that, under either requirement $O^{+} \in \partial_{\text {per }} O_{3}$ or $O_{3} \in \partial_{\text {per }} O^{+}, \bar{q}_{3}$ is negative.

We then claim:

Proposition 3.3 $\bar{p}_{1}=\bar{p}_{3}$.

Proof of Proposition 3.3 We choose now $\mathrm{O}_{2}$ so that:

$$
0 \lesseqgtr r_{2}\left(O_{2}\right) \lesseqgtr r_{2}\left(O_{3}\right)
$$

We then have:

$$
-\bar{p}_{1} \bar{q}_{2} \leq-\bar{p}_{3} \bar{q}_{2}
$$

Now that we know that $\bar{q}_{3}$ is negative, $\bar{q}_{2}$ can be taken, see Fig. 3 above, as positive or negative as we please. These two occurrences can be made compatible with the requirement $0 \lesseqgtr r_{2}\left(O_{2}\right) \lesseqgtr r_{2}\left(O_{3}\right)$. It follows that $\bar{p}_{1}=\bar{p}_{3}$, as claimed.

All the arguments above extend to the case when the periodic orbit $O^{+}$is replaced by another periodic $O_{1}$ such that $p_{1}$ is negative and $q_{1}$ is negative. By symmetry, they extend, with $p$ replacing $-q$ and $q$ replacing $-p$, to the case when $O_{3}$ (with $r_{2}\left(O_{3}\right) \lessgtr r_{2}\left(O_{1}\right)$ ) verifies $p_{3} \ngtr 0, q_{3} \gtrless 0$.

Let us now consider the case of two periodic orbits $O_{1}$ and $O_{3}$, such that $p_{1} \geq 0, q_{1} \leq 0$ and also $p_{3} \geq 0, q_{3} \leq 0$. We then claim:

Proposition 3.4 Under the assumptions above, neither $O_{3} \in \partial_{\text {per }} O_{1}$, nor $O_{1} \in \partial_{\text {per }} O_{3}$.

Proof of Proposition 7 Assume e.g., that $r_{2}\left(O_{1}\right) \geq r_{2}\left(O_{3}\right)$. Under our assumptions, $O_{1}$ is not $O^{+}$and $O_{3}$ is not $O^{-}$. Let us first assume that $p_{1} \geq 0, q_{1} \lesseqgtr 0$ and also $p_{3} \ngtr 0, q_{3} \lesseqgtr 0$.

Considering another periodic orbit $O_{2}$ such that $r_{2}\left(O_{2}\right) \gtrless r_{2}\left(O_{1}\right)$, we may take $p_{2}$ to be positive or negative as we please. Assuming that $O_{3} \in \partial_{\text {per }} O_{1}$ or $O_{1} \in \partial_{\text {per }} O_{3}$, we find, after the use of the linking inequality and the fact that $p_{2}$ can be chosen to be positive or negative, that $q_{1}=q_{3}$.

Arguing in a similar way with $r_{2}\left(O_{3}\right) \lesseqgtr r_{2}\left(O_{2}\right)$, we find that $q_{1}=q_{3}$. Then, $p_{1}-q_{1}=p_{3}-q_{3}$, a contradiction.

If now $p_{1}=0$, then, we cannot have $O_{3} \in \partial_{\text {per }} O_{1}$. Indeed, otherwise, taking $r_{2}\left(O_{2}\right) \lesseqgtr r_{2}\left(O_{3}\right)$, with $q_{2} \gtrless 0$, we find:

$$
-p_{1} q_{2}=0 \leq-p_{3} q_{2}
$$

Since $q_{2}$ is positive, $p_{3}$ is non-positive, contrary to the assumption. 
If now $O_{1} \in \partial_{\text {per }} O_{3}$, then, with $p_{1}=0$, we take $O_{2}$ such that $r_{2}\left(O_{2}\right) \gtrless r_{2}\left(O_{1}\right)$. We find that

$$
-p_{2} q_{3} \leq-p_{2} q_{1}
$$

Since $p_{2} \lesseqgtr 0,-q_{3} \geq-q_{1}$. Would this inequality be a strict inequality, then, since $p_{1}=0$ and $p_{3} \gtrless 0$, the difference of Morse indexes would be more than 1; a contradiction. Thus, $p_{3}=1, q_{3}=q_{1}$.

Furthermore, if $q_{1}=q_{3}$ is negative, then, taking $r_{2}\left(\mathrm{O}_{2}\right) \lesseqgtr r_{2}\left(\mathrm{O}_{3}\right)$, we can have $q_{2}$ negative as well as positive. This implies that $p_{1}=p_{3}=0$, a contradiction.

Thus, we must then have that $p_{1}=q_{1}=0$. There are no such orbits.

We have, under the assumption that $r_{2}\left(O_{3}\right) \leq r_{2}\left(O_{1}\right)$ settled all the cases such that such that $p_{1} \geq 0, q_{1} \lesseqgtr 0$ and also $p_{3} \gtrless 0, q_{3} \lesseqgtr 0$. By symmetry, the case $p_{1} \gtrless 0, q_{3}=0$ is also settled.

We are left with the case $p_{1}=0, q_{3}=0$.

Using the symmetry $r_{2} / r_{1}$ that transforms $p$ into $q$ and vice-versa, $q_{1}=-p_{3}$. Thus, $p_{3}-q_{3}=p_{3}=$ $0-q_{1}=p_{1}-q_{1}$, a contradiction.

Next, we claim:

Proposition 3.5 If, in all the arguments above, $r_{2}\left(O_{1}\right)=r_{2}\left(O_{3}\right)$ (that is the case when $O_{1}$ and $O_{3}$ are iterates of each other), then, either, $p_{1}=p_{3}$ or $q_{1}=q_{3}$. Therefore, $O_{1}$ and $O_{3}$ cannot be iterates of each other and this case is impossible.

Proof of Proposition 3.5 This Proposition follows from the same arguments as above.

The conclusion of the whole study above can be summarized in the following Proposition [(i) of Theorem 1.2]:

Proposition 3.6 Let $O_{1}$ and $O_{3}$ be such that $O_{3} \in \partial_{\text {per }}\left(O_{1}\right)$. Then, either, $q_{1} \lesseqgtr 0, q_{3} \lesseqgtr 0$ : we then have $p_{1}=p_{3}$ and $q_{3}=q_{1}+1$. Or $q_{1} \gtrless 0, q_{3} \gtrless 0$ : we then have $q_{1}=q_{3}$ and $p_{3}=p_{1}-1$.

Proof of Proposition 3.6 It follows from all the previous results and from the fact that $p_{3}-q_{3}=p_{1}-q_{1}-1$.

\section{Estimates on the ratio $\frac{i}{p}=\frac{2(\tilde{A}-\tilde{B})+(1)}{\tilde{A}}$ for $O^{+}$and $O_{3}$ : estimates on the homology for the first exotic contact form of J. Gonzalo and F. Varela [8]}

We have now given strong restrictions on the $\partial_{\text {per }}$ operator for the first exotic contact form of J. Gonzalo and F. Varela [8]. We would like to prove that the related homology defined by ker $\partial / \operatorname{Im} \partial$ is unbounded. Despite the strength of Proposition and the strength of the use of the linking inequalities, see above, we are not able to prove this result at this time.

However, we can prove a weaker result, see also [6, 7], stating that this homology has at least two non-zero generators for a sequence of indexes tending to infinity.

We will prove this result by two different techniques below. One of the techniques has been already used in $[6,7]$. The other one below makes more use of the arguments of the present paper and relies also on the graphs of Fig. 3. This other-more complicated proof-involves also some estimates on the ratio of indexes to the number of iterations $\frac{i}{p}=\frac{2(\tilde{A}-\tilde{B})+(1)}{\tilde{A}}$.

We revert to our earlier framework, when $O_{1}=O^{+}$. We then claim:

Proposition 4.1 Assume that, as $\bar{p}_{1}$ tends to $\infty$, we find a sequence of periodic orbits $O_{3}^{\prime}$, with $0 \lesseqgtr r_{2}\left(O_{3}^{\prime}\right) \lesseqgtr 1$, such that $O^{+} \in \partial O_{3}^{\prime}$ or $O_{3}^{\prime} \in \partial O^{+}$. Then limsup $\frac{\text { index }\left(O^{+}\right)}{\left|\bar{p}_{1}\right|} \geq 2.5$.

Proof of Proposition 4.1 We know that the index of $O^{+}$and the index of $O_{3}^{\prime}$ differ by 1 under the assumptions of the Proposition. On the other hand, the index of $O_{3}^{\prime}$ is $2\left|p_{3}-q_{3}\right|-(1)$, see [6], Sect. 7. Now, $\frac{2\left|p_{3}-q_{3}\right|}{\bar{p}_{3}}$ is equal, see [6], Sect. 7, to $\frac{2|\tilde{A}-\tilde{B}|}{|\tilde{A}|}\left(O_{3}^{\prime}\right)$. We know, since $\bar{p}_{1}$ is negative that $\bar{p}_{3}$ is negative, so that $\tilde{A}\left(O_{3}^{\prime}\right)$ is negative. Fig. 3 above allows to conclude. 
Before proving (ii) of Theorem 1.2, we need three more Propositions. We will provide two distinct proofs of (ii) of Theorem 1.2.

We start by proving Proposition 4.2 that refines the previous proposition and that reads:

Proposition 4.2 Assume that, as $\bar{p}_{1}$ tends to infinity, there is a sequence of periodic orbits $O_{3}^{\prime}$, with $0 \lessgtr$ $r_{2}\left(O_{3}^{\prime}\right) \lesseqgtr 1$, such that $O^{+} \in \partial O_{3}^{\prime}$. Then, limsup $\frac{\operatorname{index}\left(O^{+}\right)}{\left|\bar{p}_{1}\right|} \geq 3.2$.

Proof of Proposition 4.2 Since $O^{+} \in \partial O_{3}^{\prime}$, the critical level of $O_{3}^{\prime}$ is larger than the critical level of $\mathrm{O}^{+}$.

The critical value of $O_{3}^{\prime}$ is, see [6], Sect. 7:

$$
-2 \pi\left|\bar{p}_{3}\right| \frac{\left(A \tilde{B} r_{1}+B \tilde{A} r_{2}\right)}{\tilde{A}}\left(O_{3}^{\prime}\right)
$$

Indeed, this critical value is, by [6], Sect. 7:

$$
\pi \times 2\left(\bar{p}_{3}-\bar{q}_{3}\right) \times \frac{\left(A \tilde{B} r_{1}+B \tilde{A} r_{2}\right)}{\tilde{A}-\tilde{B}}\left(O_{3}^{\prime}\right)
$$

which we rewrite (observe that $\tilde{A}\left(O_{3}^{\prime}\right) \lesseqgtr 0$ ):

$$
\left|\bar{p}_{3}\right| 2 \pi\left(\left(\bar{p}_{3}-\bar{q}_{3}\right) \frac{\left(A \tilde{B} r_{1}+B \tilde{A} r_{2}\right)}{\left|\bar{p}_{3}\right|(\tilde{A}-\tilde{B})}\left(O_{3}^{\prime}\right)\right)=-2 \pi\left|\bar{p}_{3}\right|\left(\frac{\left(A \tilde{B} r_{1}+B \tilde{A} r_{2}\right)}{\tilde{A}}\left(O_{3}^{\prime}\right)\right)
$$

For $O^{+}$, by direct computation, see the formulae for $\xi_{0}$ and $\alpha_{1}$ in [6], the critical value is:

$$
\left|\bar{p}_{1}\right| \frac{2 \pi}{\sqrt{2}}=\sqrt{2} \pi\left|\bar{p}_{1}\right|=\sqrt{2} \pi\left|\bar{p}_{3}\right|
$$

Observe now that:

$$
-2 \pi\left|\bar{p}_{3}\right|\left(\frac{\left(A \tilde{B} r_{1}+B \tilde{A} r_{2}\right)}{\tilde{A}}\left(O^{+}\right)\right)=2 \pi\left|\bar{p}_{3} B\right|=\pi \sqrt{2}\left|\bar{p}_{3}\right|
$$

When comparing the critical values, we can therefore compare the values of the function:

$$
-\frac{\left(A \tilde{B} r_{1}+B \tilde{A} r_{2}\right)}{\tilde{A}}
$$

at the curves $O^{+}$and $O_{3}^{\prime}$. There should be larger at $O_{3}^{\prime}$, with $\tilde{A}_{3}\left(O_{3}^{\prime}\right) \lesseqgtr 0$.

Looking up Fig. 3 above, this implies that $r_{2} \leq .648$. Then $\left|\frac{i}{p}\right|=-2 \frac{\tilde{A}-\tilde{B}}{\tilde{A}}$ is larger than 3.2 as claimed. The conclusion follows.

Next, and this will allow us to give another proof of (ii) of Theorem 1.2, we have the following Proposition that gives the $H_{0}^{1}$-index of the periodic orbits corresponding to $r_{1}=0$ or $r_{2}=0$ :

Proposition 4.3 The v-rotation on the simple orbits corresponding to $r_{1}=0$ or $r_{2}=0$ is at least $7 \pi$. Therefore, the index of the iterate of order $\bar{p}, i_{\bar{p}}$, is at least $7 \bar{p}$.

Proof of Proposition 4.3 We consider neighboring periodic orbits to the simple periodic orbit $O_{0}$ corresponding to $r_{1}=0$. It is not very difficult to see that $O_{0}$ is elliptic, this involves the computation of the linearized operator at $O_{0}$; it is a long, but straightforward computation of the quantity $\tau$, see [1], p 2, [4] p 21, involved in the formula of the linearized operator $\ddot{\eta}+\eta \tau$.

The neighboring periodic orbits have associated numbers $(p, q)$ that tend both to $-\infty$ as $r_{1}$ tends to zero: the ratio $\frac{\tilde{A}}{\tilde{B}}$ is irrational at $r_{1}=0$.

$p$ is the number of counter-clockwise rotations in the "surviving" $\left(x_{3}, x_{4}\right)$-plane. We thus may consider our neighboring periodic orbits as made of $p$ distinct pieces of nearly closed $\xi_{0}$-pieces of orbits. Each of this distinct piece converges to the periodic orbit $O_{0}$ as $r_{1}$ tends to zero. 
We consider some base point $x_{0}$ on $O_{0}$. We pick up $v$ at $x_{0}$, equal therefore to $v\left(x_{0}\right)$ and we $\xi_{0}$-transport it around the periodic orbit $O_{0}$ over $p$-revolutions. This transported vector is denoted $u=u(s)$, where $s$ is the running parameter over the periodic orbit $O_{0}$, based at $x_{0}$ and iterated an infinite number of times. Over each of these $p \xi$-pieces, $u(s)$ will coincide with $v$ a certain number of times. This number of times can be $n$ or $n-1$, where $n$ is the $H_{0}^{1}$-index of $O_{0}$, with no base point assigned, that is, starting from any point of $O_{0}$, $v$ turns more than $n \pi$ and less than $(n+1) \pi$ over $O_{0}$.

On the approaching $\xi_{0}$-orbits, we can take a base point close to $x_{0}$ and define a $\xi_{0}$ transported vector $\hat{u}(s)$, equal to $v$ at the base point. Using continuity, $v$ will coincide, on each of the $p$-pieces of $\xi$-orbit with $\hat{u}(s)$ at most $n$-times. It follows that on the whole approaching $\xi_{0}$ periodic orbit, $v$ will coincide with the transported vector $\hat{u}(s)$ at most $p n$-times. The index of this periodic orbit is then less than or equal to $p n+1$, since it is less than or equal to $p n$ under the constraint that the variation of the curve is along $v$ at the base point.

Thus, the ratio of the index $i_{p}$ to $p$ is less than or equal to $\frac{p n+1}{p}$. It is therefore less than or equal to $n$ as $p$ tends to infinity. This ratio is equal to $\frac{-2(\tilde{A}-\tilde{B})}{\tilde{A}}$ at the periodic orbit. This ratio is $2 \pi$ at $O_{0}$. It follows that $n$ is larger than 6 . The claim follows.

The last Proposition needed for the proof of (ii) of Theorem 1.2 is about the number of hyperbolic orbits of $\xi_{0}$ of index $2 k$. It reads:

Proposition 4.4 Let $H_{2 k}$ be the set of hyperbolic periodic orbits of index $2 k$ of $\xi_{0}$ and let $n_{k}$ be its cardinal. Then, $n_{k-1}+4 \geq n_{k} \geq n_{k-1}+2$ as $k$ tends to infinity.

Proof of Proposition 4.4 For $r_{2} \geq \frac{1}{2}$, we consider the ratio of the index $i$ to the number $q$. This ratio is equal to $\frac{-2(\tilde{A}-\tilde{B})}{\tilde{A}}$. The graph of this function is in Fig. 3. The minimum $m$ of this function on $\left[\frac{1}{2}, 1\right]$ is strictly larger than 1 and strictly less than 2 .

It follows that if $\frac{i}{q+1} \leq m \leq \frac{i}{q}$, then $\frac{i+2}{q+3} \leq \frac{i}{q+1} \leq m \leq \frac{i}{q} \leq \frac{i+2}{q+1}$ for $p$ or $q$ large enough. There is at least one more hyperbolic orbit in $H_{i+2}$ with respect to $H_{i}$ in the $r_{2}$-interval $\left[\frac{1}{2}, 1\right]$, maybe 2 . The claim follows using the symmetry between $r_{1}$ and $r_{2}$.

We are now ready to prove (ii) of Theorem 1.2; we will provide two proofs.

Proof of (ii) of Theorem 1.2 We consider the periodic orbits of prescribed index $i$. This set is denoted $C_{i}$. $C_{i}$ is made of two subsets. To see this, we first consider the odd index $(2 k-1)$. Then $C_{2 k-1}$ is made of the hyperbolic orbits of index $(2 k-1)$ and of the iterates of the elliptic orbits $O_{0}$ and $O_{1}$ (corresponding to $r_{1}=0$ and $r_{2}=0$, respectively). The set of hyperbolic orbits of index $(2 k-1)$ is in one to one correspondence with the set $H_{2 k}$ of periodic orbits of index $2 k$ introduced earlier. The iterates of $O_{0}$ and $O_{1}$ have a strictly increasing index since the $v$-rotation on each of them is larger than $3 \pi$, so that their index is at least three. Therefore, there are either two iterates contributing to the index $i$ or none.

Thus, $C_{2 k-1}$ is made of $H_{2 k-1}$ that has as many elements as $H_{2 k}$ and of $K_{2 k-1}$, that is empty or has two elements which are iterates of $O_{0}$ and $O_{1}$.

The same conclusion applies to $C_{2 k}$.

By Corollary 1 of [6], the intersection operator from $H_{2 k}$ to $H_{2 k-1}$ is zero. Furthermore, by Proposition 4.3, there must be an infinite numbers of intervals of iterations $\left[p_{m}, p_{m}+5\right]$ where the $C_{j}=\emptyset$ for $j \in\left[p_{m}, p_{m}+5\right]$. Considering an odd index $(2 l-1)$ in this interval, such that $2 l$ and $(2 l-2)$ are also in this interval, the claim of (ii) of Theorem 1.2 follows now from Proposition 4.4 and the fact that the intersection operator from $C_{2 l}=H_{2 l}$ into $C_{2 l-1}=H_{2 l-1}$ is zero.

There is a slightly more difficult proof of this result that does not use the full strength of Proposition 4.3, only that the index of the iterates of $O_{0}$ nd $O_{1}$ is strictly increasing. This proof relies more on Propositions 4.1 and 4.2.

Using Proposition 4.2, we know that either no periodic orbit with $0 \lesseqgtr r_{1} \lesseqgtr 1$ will have an iterate of one of the elliptic orbits in its range as the index tends to infinity, or there are infinitely many intervals $\left[p_{m}, p_{m}+3\right]$ such that no iterate of the elliptic orbits $O^{ \pm}$is in $C_{j}$ for $j \in\left[p_{m}, p_{m}+3\right]$. These sets reduce then to $H_{j}$.

Under the first occurrence, we know, by Proposition 4.1, that there are infinitely many intervals $\left[p_{m}, p_{m}+2\right]$ such that no iterate of the elliptic orbits $O^{ \pm}$is in $C_{j}$ for $j \in\left[p_{m}, p_{m}+2\right]$.

If $p_{m}=(2 k)$, then the intersection operator is zero from $H_{2 k}$ into $H_{2 k-1} \cdot H_{2 k-1}$ has two more elements than $H_{2 k-2}$ and the intersection operator, under our assumption, maps $H_{2 k-1}$ into $H_{2 k-2}$. The conclusion of (ii) of Theorem 1.2 follows if this occurrence repeats infinitely many times. 
If, on the other hand, $p_{m}=(2 k+1)$, then the homology is non-zero at the order $(2 k+1)$ unless two elements of $H_{2 k+1}$ are in the range, for the intersection operator, of two iterates of the elliptic orbits that must contribute to $C_{2 k+2}$.

The argument repeats for each large enough odd index $(2 j+1)$ : if the homology is zero, $C_{2 j+1}$ reduces to $H_{2 j+1}$ whereas $C_{2 j+2}$ must have two additional elements coming from the elliptic orbits. This contradicts then Proposition 4.2 and concludes the proof of (ii) of Theorem 1.2 under the assumption that no hyperbolic orbit will have an iterate of one of the elliptic orbits in its range for large enough indexes.

If we assume now that a sequence of periodic orbits with $0 \leq r_{1} \lesseqgtr 1$, with indexes going to infinity, have iterates of the two elliptic orbits in their range, then, as stated above, there are infinitely many intervals $\left[p_{m}, p_{m}+3\right]$ such that no iterate of the elliptic orbits $O^{ \pm}$is in $C_{j}$ for $j \in\left[p_{m}, p_{m}+3\right]$. These sets reduce then to $H_{j}$.

The argument is then somewhat similar to the argument above.

We, again, distinguish the case when $p_{m}+3=2 k$ and the case when $p_{m}+3=2 k+1$.

If $p_{m}+3$ is $2 k$, we use the fact, Corollary 1 of [6], that the intersection map from $H_{2 k}$ to $H_{2 k-1}$ is zero. On the other hand, by Proposition 4.4 above, $H_{2 k-1}$ has at least two more elements then $C_{2 k-2}=H_{2 k-2}$. The conclusion of (ii) of Theorem 1.2 follows then if this repeats for infinitely many indexes $p_{m}+3=2 k$.

If $p_{m}+3=2 k+1$, then $C_{p_{m}+3}=H_{p_{m}+3}=H_{2 k+1}$, which has at least two more elements then $C_{p_{m}+2}=H_{p_{m}+2}=H_{2 k}$. The homology is then non-zero for the index $(2 k+1)$, unless two elements of $H_{2 k+1}$ are in the range, for the intersection operator of the elliptic orbits of index $(2 k+2)$ (they cannot be in the range of the intersection operator restricted to $\mathrm{H}_{2 k+2}$, by Corollary 1 of [6]). If this happens, if the homology is zero, then all of $H_{2 k}$ is in the range of the intersection operator. Moving then to $C_{2 k-1}=H_{2 k-1}$, this has two more elements to the least than $H_{2 k-2}$; but $C_{2 k-2}$ might have two more elements coming from the iterates of the elliptic orbits. It is only under this additional occurrence that the homology at the order $(2 k-1)$ might be zero. Furthermore, we then know that all of $C_{2 k-2}$ is in the range of the intersection operator. The argument then moves to $C_{2 k-3}$ and $C_{2 k-4}$. If the homology at the order $(2 k-3)$ is zero, $C_{2 k-3}$ should be reduced to $H_{2 k-3}$ and all of $C_{2 k-4}$ is in the range of the intersection operator, implying that two iterates of the elliptic orbit contribute there. A decreasing induction starts then, implying that, at least for large indexes less than $(2 k-1), C_{2 l-1}=H_{2 l-1}$ whereas $C_{2 l-2}$ has two more elements than $H_{2 l-2}$. This contradicts Proposition 4.2, as there are infinitely many intervals $\left[p_{m}, p_{m}+3\right]$ such that no iterate of the elliptic orbits $O^{ \pm}$is in $C_{j}$ for $j \in\left[p_{m}, p_{m}+3\right]$. The claim of (ii) of Theorem 1.2 follows.

Open Access This article is distributed under the terms of the Creative Commons Attribution License which permits any use, distribution, and reproduction in any medium, provided the original author(s) and the source are credited.

\section{References}

1. Bahri, A.: Pseudo-orbits of contact forms. Pitman Research Notes in Mathematics Series No. 173, Scientific and Technical, London (1988)

2. Bahri, A.: Un problème variationnel sans compacité en géométrie de contact. C. R. Acad. Sci. Paris 299 Ser. I 15, pp. 757-760 (1984)

3. Bahri, A.: Classical and quantic periodic motions of multiply polarized spin-manifolds. Pitman Research Notes in Mathematics Series No. 378, Longman and Addison-Wesley, London and Reading, MA (1998)

4. Bahri, A.: Flow-lines and algebraic invariants in contact form geometry. PNLDE, vol. 53, Birkhauser, Boston (2003)

5. Bahri, A.: Compactness. Adv. Nonlinear Stud. 8(3), 465-568 (2008)

6. Bahri, A.: On the contact homology of the first exotic contact form/structure of J. Gonzalo and F. Varela. Arab. J. Math. 3. doi:10.1007/s40065-014-0097-2 (this issue)

7. Bahri, A.: Morse relations and Fredholm deformations of $v$-convex contact forms. Arab. J. Math. 3. doi:10.1007/s40065-0140098-1 (this issue)

8. Gonzalo, J.; Varela, F.: Modèles globaux des variétés de contact. Third Schnepfenried geometry conference Astérisque No. 107-108, vol. 1, pp. 163-168. SMF Publications, Paris (1983) 